\title{
A New Family of Semicircular Models: The Semicircular Laplace Distributions
}

\author{
Byoung Jin $\mathrm{Ahn}^{1)}$, Hyoung-Moon Kim²)
}

\begin{abstract}
It is developed that a family of the semicircular Laplace distributions for modeling semicircular data by simple projection method. Mathematically it is simple to simulate observations from a semicircular Laplace distribution. We extend it to the $l$-axial Laplace distribution by a simple transformation for modeling any arc of arbitrary length. Similarly we develop the $l$-axial log-Laplace distribution based on the log-Laplace distribution. A bivariate version of $l$-axial Laplace distribution is also developed.
\end{abstract}

Keywords: l-axial data; heavy tail; projection; double exponential.

\section{Introduction}

Full circular models are prevalent at most textbooks (Fisher, 1993; Jammalamadaka and SenGupta, 2001; Mardia and Jupp, 2000). However those are not always necessary since sometimes angular data are given as modulo $\pi$. Guardiola (2004) and Jones (1968) noted this fact.

For example, when an aircraft is lost but its point of departure and its initial headings are known, a random variable having values on a semicircle well suffice for such a problem. Similarly, when a sea turtle emerges from the ocean in search of a nesting site on dry land, a semicircular random variable is sufficient for modeling such directional data. Third example is concerned with the canting angles of falling raindrops (Ugai et al., 1977): raindrops are shaped roughly like rugby balls and tend to fall at an angle because of wind resistance and turbulence. These data are called axial or semicircular data.

Guardiola (2004) obtained the semicircular normal distribution using a simple projection. Similarly we project a Laplace distribution over a semicircular segment to obtain the semicircular Laplace(SCL) distribution. Laplace probability density function(pdf) is in-between two $p d f \mathrm{~s}$ : a normal $p d f$ and a $t p d f$. Similarly a semicircular Laplace $p d f$ is located between two $p d f \mathrm{~s}$ : a semicircular $\operatorname{Normal}(\mathrm{SCN}) p d f$ and a semicircular $t(\mathrm{SCT})$ $p d f(\operatorname{Kim}, 2008)$.

1) Professor, Department of Applied Statistics, Konkuk University, 1 Hwayang-Dong, Gwangjin-Gu, Seoul 143-701, Korea

2) Associate Professor, Department of Applied Statistics, Konkuk University, 1 Hwayang-Dong, Gwangjin-Gu, Seoul 143-701, Korea. Correspondence: hmkim@konkuk.ac.kr 
This paper is organized as follows. Section 2 defines the distribution, lists some of its basic properties and derives the trigonometric moments. Furthermore we consider the asymptotic behavior of the SCL distribution. We estimate parameters of the SCL distribution by a maximum likelihood method in Section 3. We concludes in Section 4 with extensions of the SCL distribution: the $l$-axial Laplace distribution for modeling any arc of arbitrary length, say $2 \pi / l, l=1,2, \ldots$, and the bivariate version of $l$-axial Laplace distribution. Similarly it is developed that the $l$-axial log-Laplace distribution based on the (linear) log-Laplace distribution. 'linear' is used for distinguishing circular distribution from usual distribution on the real line.

\section{Semicircular Laplace Distribution}

\subsection{Definition and some basic properties}

The SCL distribution is obtained by projecting a Laplace distribution over a semicircular segment. Let $X$ have a Laplace distribution with $\sigma$, i.e., the density of $X$ is

$$
\frac{1}{2 \sigma} e^{-\frac{|x|}{\sigma}}, \quad-\infty<x<\infty, \sigma>0 .
$$

For brevity, we shall say that $X$ is Laplace $(0, \sigma)$. This is also known as the Double Exponential distribution. For a positive real number $r$, define the angle $\theta$ by $\theta=\tan ^{-1}(x / r)$. Hence, $x=r \tan (\theta)$. Obviously, the $p d f$ of $\theta$ is given by

$$
\frac{1}{2 \varphi} \sec ^{2}(\theta) \exp \left(-\frac{|\tan (\theta)|}{\varphi}\right), \quad \varphi=\frac{\sigma}{r}, \quad-\frac{\pi}{2}<\theta<\frac{\pi}{2} .
$$

More generally, we introduce the parameter $\mu$ as the location parameter for the SCL distribution and write the $p d f$ as

$$
\frac{1}{2 \varphi} \sec ^{2}(\theta-\mu) \exp \left(-\frac{|\tan (\theta-\mu)|}{\varphi}\right), \quad-\frac{\pi}{2}+\mu<\theta<\frac{\pi}{2}+\mu, \quad-\pi<\mu<\pi .
$$

Then, we say that $\theta$ is an SCL random variable with parameters $\mu$ and $\varphi$; for brevity, we shall also say that $\theta$ is $\operatorname{SCL}(\mu, \varphi)$. Geometrically $r$ is the distance between the radius and the support of the Laplace density. The closer the support is to the radius, the larger $\varphi$.

Figure 2.1 shows the shape of $(2.3)$ for three values of $\sigma(=1,2,5)$ with $r^{2}=10$ and $\mu=0$. It is straightforward to generate samples from an SCL distribution. First, generate samples from a Laplace distribution and then use the inverse transformation, $\theta=\mu+\tan ^{-1}(x / r), r=\sigma / \varphi$. Similarly, the cumulative distribution function(cdf) of an SCL distribution is $F(\theta ; \mu, \varphi)=L(r \tan (\theta-\mu))$, where the function $L(\cdot)$ is a $c d f$ of a Laplace distribution, Laplace $(0, \sigma)$, i.e.,

$$
L(x)=0.5\left[1+\operatorname{sgn}(x)\left\{1-\exp \left(-\frac{|x|}{\sigma}\right)\right\}\right] .
$$




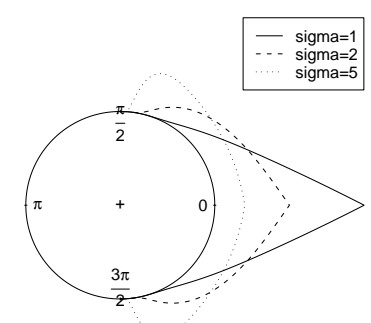

Figure 2.1: The probability density functions $\operatorname{SCL}(0, \varphi)$, where $\varphi=\sigma / r$ and $r^{2}=10$

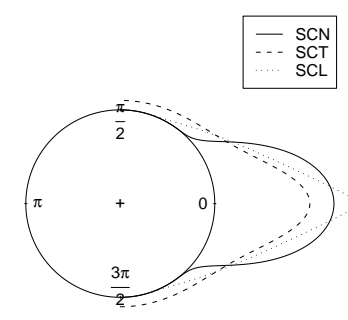

Figure 2.2: An SCN $p d f$, an SCT $p d f$ and an SCL $p d f$

The sign function, $\operatorname{sgn}(x)$, is a logical function which extracts the sign of a real number. So

$$
\operatorname{sgn}(x)=\left\{\begin{aligned}
1, & \text { if } x>0 \\
0, & \text { if } x=0 \\
-1, & \text { if } x<0
\end{aligned}\right.
$$

To compare the tail behavior of an SCN distribution, an SCT distribution and an SCL distribution, Figure 2.2 shows three $p d f \mathrm{~s}$. We use the same transformation, $x=$ $r \tan (\theta)$ and the same $r^{2}=10$ to get all three $p d f \mathrm{~s}$ for comparison purpose. An SCN distribution is obtained from transforming the standard normal distribution (Guardiola, 2004). Similarly an SCT is derived from transforming $t$ distribution with $d f=1$ (Kim, 2008). An SCL $p d f$ is the $p d f$ of Figure 2.1 with $\sigma=1$. From the Figure 2.2, we note that an SCT distribution has more heavier tail than an SCN distribution. Similar relationship also appears at linear statistics, i.e. a relationship between a normal distribution and a $t$ distribution. Furthermore an SCL $p d f$ is located between the two $p d f \mathrm{~s}$ which is also true 
for a Laplace $p d f$, i.e. a Laplace $p d f$ is located between a Normal $p d f$ and a $t p d f$.

The following properties of the density (2.3) follow immediately from the definition of an SCL distribution and the properties of a Laplace distribution. Suppose $\theta_{1} \sim$ $\operatorname{SCL}(\mu, \varphi)$ independently of $\theta_{2} \sim \operatorname{SCL}(\mu, \varphi)$, then

$$
\text { (a) }\left|r \tan \left(\theta_{1}-\mu\right)\right| \sim \frac{\sigma}{2} \chi^{2}(2) \text { and (b) } \frac{\left|r \tan \left(\theta_{1}-\mu\right)\right|}{\left|r \tan \left(\theta_{2}-\mu\right)\right|} \sim F(2,2),
$$

where $\chi^{2}(\nu)$ denotes a $\chi^{2}$ distribution with $\nu$ degree of freedom(df) and $F\left(\nu_{1}, \nu_{2}\right)$ represents a $F$ distribution with $\nu_{1}$ and $\nu_{2} d \mathrm{fs}$.

We consider the asymptotic behavior of the SCL distribution when $\varphi \rightarrow 0$. Suppose $\theta$ follows $\operatorname{SCL}(\mu, \varphi)$. Let $y=(\theta-\mu) / \varphi$ and then use the change of variable technique. For sufficiently small $\varphi$, we have $\tan (y \varphi) \simeq y \varphi$ and $\sec (y \varphi) \simeq 1$ by the first order approximation of the Taylor series expansion. Hence, the distribution of $\theta$ becomes Laplace $(\mu, \varphi)$. So, for sufficiently small $\varphi$, the SCL distribution can be approximated by a (linear) Laplace distribution.

\subsection{Trigonometric moments}

Similar to those of any circular density, trigonometric moments of the SCL distribution are defined as follows: $\phi_{p}=E e^{i p \theta}=\alpha_{p}+i \beta_{p}=E \cos (p \theta)+i E \sin (p \theta), p=$ $0, \pm 1, \pm 2, \cdots$. We assumed that the location parameter $\mu=0$ without loss of generality.

Theorem 2.1 Under the $p d f(2.3)$ with $\mu=0$, the first four $\alpha_{p}=E \cos (p \theta), p=$ $1,2,3,4$, are given as follows:

$$
\begin{aligned}
& \alpha_{1}=\frac{\pi}{2 \varphi}\left\{H_{0}\left(\frac{1}{\varphi}\right)-Y_{0}\left(\frac{1}{\varphi}\right)\right\}, \\
& \alpha_{2}=\frac{\sqrt{\pi}}{\sqrt{2} \varphi^{\frac{3}{2}}}\left\{H_{-\frac{1}{2}}\left(\frac{1}{\varphi}\right)-Y_{-\frac{1}{2}}\left(\frac{1}{\varphi}\right)\right\}-1, \\
& \alpha_{3}=\frac{\pi}{2 \varphi^{2}}\left\{Y_{-1}\left(\frac{1}{\varphi}\right)-H_{-1}\left(\frac{1}{\varphi}\right)\right\}-\frac{3}{\pi \varphi} G_{13}^{31}\left(\frac{1}{4 \varphi^{2}} \mid \begin{array}{c}
-1 / 2 \\
0,0,1 / 2
\end{array}\right) \text {, } \\
& \alpha_{4}=8\left\{1-\frac{1}{2 \sqrt{\pi} \varphi} G_{13}^{31}\left(\frac{1}{4 \varphi^{2}} \mid \begin{array}{c}
-1 / 2 \\
1 / 2,0,1 / 2
\end{array}\right)\right\},
\end{aligned}
$$

where $H_{\nu}(z)$ is the Struve function, $Y_{\nu}(z)$ is the Bessel function of the second kind (Abramowitz and Stegun, 1972) and $G_{p q}^{m n}\left(x \mid \begin{array}{l}a_{1}, \ldots, a_{p} \\ b_{1}, \ldots, b_{q}\end{array}\right)$ is called as Meijer's G-function (Gradshteyn and Ryzhik, 2007). Furthermore, $\beta_{p}=E \sin (p \theta), p=0, \pm 1, \pm 2, \ldots$, are 0 .

Proof: The proof is the process of using some transformations. For the first cosine moment, use the transformation $x=\tan (\theta)$ and then use the property of an even function. So

$$
\alpha_{1}=\frac{1}{\varphi} \int_{0}^{\infty}\left(1+x^{2}\right)^{-\frac{1}{2}} e^{-\frac{x}{\varphi}} d x
$$


The result $\alpha_{1}$ follows by the integral formula 3.387.7 (Gradshteyn and Ryzhik, 2007). To obtain $\alpha_{2}$, we use the transformation $x=\tan (\theta)$ followed by using the properties of an even function and a $p d f$. So

$$
\alpha_{2}=\frac{2}{\varphi} \int_{0}^{\infty}\left(1+x^{2}\right)^{-1} e^{-\frac{x}{\varphi}} d x-1
$$

The result is immediate by the same integral formula of $\alpha_{1}$. For the third cosine moment, use the transformation $x=\tan (\theta)$ and then use the property of an even function.

$$
\alpha_{3}=\frac{1}{\varphi} \int_{0}^{\infty}\left(1+x^{2}\right)^{-\frac{3}{2}} e^{-\frac{x}{\varphi}} d x-\frac{3}{\varphi} \int_{0}^{\infty} x^{2}\left(1+x^{2}\right)^{-\frac{3}{2}} e^{-\frac{x}{\varphi}} d x .
$$

For the first integral of the right hand side, we can use the same integral formula of $\alpha_{1}$. Furthermore, by the integral formula 3.389.2 (Gradshteyn and Ryzhik, 2007), we can solve the second integral of the right hand side. To get the fourth cosine moment, first use the transformation $x=\tan (\theta)$ followed by the property of an even function, then

$$
\alpha_{4}=8\left\{1-\frac{1}{\varphi} \int_{0}^{\infty} x^{2}\left(1+x^{2}\right)^{-2} e^{-\frac{x}{\varphi}} d x\right\} .
$$

The result follows using by the integral formula 3.389.2 (Gradshteyn and Ryzhik, 2007) again. Note that like any other symmetric circular density, $\beta_{p}=E \sin (p \theta)$, are 0 as the density is symmetric about 0 .

The Struve function has a power series expansion

$$
H_{\nu}(z)=\left(\frac{z}{2}\right)^{\nu+1} \Sigma_{k=0}^{\infty} \frac{(-1)^{k}\left(\frac{z}{2}\right)^{2 k}}{\Gamma\left(k+\frac{3}{2}\right) \Gamma\left(k+\nu+\frac{3}{2}\right)} .
$$

The Bessel functions of the second kind are solutions of the Bessel differential equation. They are singular(infinite) at the origin $(z=0) . Y_{\nu}(z)$ is also called the Neumann function. The Meijer's G-function, $G_{p q}^{m n}\left(x \mid \begin{array}{c}a_{1}, \ldots, a_{p} \\ b_{1}, \ldots, b_{q}\end{array}\right)$, satisfies the following linear $q^{t h}$-order differential equation:

$$
\left[(-1)^{p-m-n} x \Pi_{j=1}^{p}\left(x \frac{d}{d x}-a_{j}+1\right) \Pi_{j=1}^{q}\left(x \frac{d}{d x}-b_{j}\right)\right] y=0, \quad[p \leq q] .
$$

\section{Parameter Estimation}

The minus log-likelihood for a random sample of size $n, \theta=\left(\theta_{1}, \ldots, \theta_{n}\right)$, from the SCL distribution (2.3) is given by

$$
-l(\mu, \varphi ; \theta)=n \log (2 \varphi)+\Sigma_{i=1}^{n} \log \left(\cos ^{2}\left(\theta_{i}-\mu\right)\right)+\Sigma_{i=1}^{n} \frac{\left|\tan \left(\theta_{i}-\mu\right)\right|}{\varphi} .
$$

To find maximum likelihood estimates, we can use any minimization subroutine for direct minimization of the minus log-likelihood itself. 


\section{Extensions}

We extend the suggested model to the $l$-axial distribution, which is applicable to any arc of arbitrary length say $2 \pi / l$ for $l=1,2, \cdots$. Occasionally, measurements result in any arc of arbitrary length, say $2 \pi / l, l=1,2, \ldots$, so it is desirable to extend the SCL distribution. To construct the $l$-axial Laplace distribution, we consider the $p d f(2.2)$ and use the transformation $\theta^{*}=2 \theta / l, l=1,2, \cdots$. The $p d f$ of $\theta^{*}$ is given by

$$
\frac{l}{4 \varphi} \sec ^{2}\left(\frac{l \theta^{*}}{2}\right) \exp \left(-\frac{\left|\tan \left(\frac{l \theta^{*}}{2}\right)\right|}{\varphi}\right), \quad \varphi=\frac{\sigma}{r}, \quad-\frac{\pi}{l}<\theta^{*}<\frac{\pi}{l} \text {. }
$$

Note that when $l=2$, the $p d f(4.1)$ is the same as the $p d f(2.2)$, the SCL $p d f$. When $l=1$, it becomes the $p d f$ of a circular Laplace distribution.

If $X$ has a Laplace $(0, \sigma)$, then $Y=e^{X}$ has a $\log$-Laplace distribution with the same parameters. So, using $y=r \tan (\theta)$, we get a $p d f$,

$$
\frac{1}{\sigma \sin (2 \theta)} \exp \left(-\frac{|\log (r \tan (\theta))|}{\sigma}\right), \quad 0<\theta<\frac{\pi}{2} .
$$

Suppose $\theta^{*}=4 \theta / l, l=1,2, \ldots$, then $\theta^{*}$ has a $p d f$,

$$
\frac{l}{4 \sigma \sin \left(\frac{l \theta^{*}}{2}\right)} \exp \left(-\frac{\left|\log \left(r \tan \left(\frac{l \theta^{*}}{4}\right)\right)\right|}{\sigma}\right), \quad 0<\theta^{*}<\frac{2 \pi}{l} .
$$

We say that $\theta^{*}$ follows the $l$-axial $\log$-Laplace distribution. When $l=1$, we name it as a circular $\log$-Laplace distribution and $l=2$, we call it as a semicircular log-Laplace distribution. (4.2) is the derived distribution when $l=4$ and has a support $0<\theta<\pi / 2$.

We can construct a bivariate SCL distribution in a manner similar to the construction of a univariate SCL distribution. We shall use the same semicircular transformation applied in a bivariate context. The density function of the bivariate SCL distribution is defined as

$$
\frac{\sec ^{2}\left(\theta_{1}\right) \sec ^{2}\left(\theta_{2}\right)}{\pi \varphi^{2} \sqrt{1-\rho^{2}}} K_{0}\left(\frac{\sqrt{2} q^{*}}{\varphi}\right), \quad \varphi=\frac{\sigma}{r}, \quad-\frac{\pi}{2}<\theta_{i}<\frac{\pi}{2}, i=1,2,
$$

where $q^{*}=\sqrt{\left\{\tan ^{2}\left(\theta_{1}\right)-2 \rho \tan \left(\theta_{1}\right) \tan \left(\theta_{2}\right)+\tan ^{2}\left(\theta_{2}\right)\right\} /\left(1-\rho^{2}\right)}$ and $K_{\lambda}(\cdot)$ is a modified Bessel function of the second kind (Abramowitz and Stegun, 1972).

To construct this density, we begin with the bivariate Laplace density (Johnson and Kotz, 1972)

$$
f\left(x_{1}, x_{2}\right)=\frac{1}{\pi \sigma^{2} \sqrt{1-\rho^{2}}} K_{0}\left(\frac{\sqrt{2} q}{\sigma}\right), \quad-\infty<x_{i}<\infty, i=1,2,
$$


where $\sigma>0$ and $q=\sqrt{\left(x_{1}^{2}-2 \rho x_{1} x_{2}+x_{2}^{2}\right) /\left(1-\rho^{2}\right)}$. Consider the transformation $x_{i}=$ $r \tan \left(\theta_{i}\right), i=1,2$. The Jacobian is $J=r^{2} \sec ^{2}\left(\theta_{1}\right) \sec ^{2}\left(\theta_{2}\right)$. Consequently, the $p d f$ of a bivariate SCL distribution is obtained using simple algebra. Note that (4.5) has a slightly different marginal $p d f$ form from $(2.1)$ as follows:

$$
f\left(x_{1}\right)=\frac{1}{\sqrt{2} \sigma} \exp \left(-\frac{\sqrt{2}\left|x_{1}\right|}{\sigma}\right), \quad-\infty<x_{1}<\infty, \sigma>0 .
$$

Similar to the method of constructing(univariate) $l$-axial Laplace distribution, it is simple to construct bivariate $l$-axial Laplace distribution. Let $\theta_{i}^{*}=2 \theta_{i} / l, i=1,2$ and $l=$ $1,2, \ldots$, then the joint $p d f$ of $\boldsymbol{\theta}^{*}=\left(\theta_{1}^{*}, \theta_{2}^{*}\right)^{t}$ is given by

$$
\frac{l^{2} \sec ^{2}\left(\frac{l \theta_{1}^{*}}{2}\right) \sec ^{2}\left(\frac{l \theta_{2}^{*}}{2}\right)}{4 \pi \varphi^{2} \sqrt{1-\rho^{2}}} K_{0}\left(\frac{\sqrt{2} q^{* *}}{\varphi}\right), \quad-\frac{\pi}{l}<\theta_{i}<\frac{\pi}{l}, i=1,2 \text { and } l=1,2, \ldots,
$$

where $q^{* *}=\sqrt{\left\{\tan ^{2}\left(l \theta_{1}^{*} / 2\right)-2 \rho \tan \left(l \theta_{1}^{*} / 2\right) \tan \left(l \theta_{2}^{*} / 2\right)+\tan ^{2}\left(l \theta_{2}^{*} / 2\right)\right\} /\left(1-\rho^{2}\right)}$. When $l=1$, we call it as bivariate circular Laplace distribution. If $l=2,(4.7)$ give the same $p d f$ as (4.4), the bivariate SCL distribution.

\section{References}

Abramowitz, M. and Stegun, I. A. (1972). Handbook of Mathematical Functions: with Formulas, Graphs, and Mathematical Tables, Dover Publications, New York.

Fisher, N. I. (1993). Statistical Analysis of Circular Data, Cambridge University Press, Cambridge.

Gradshteyn, I. S. and Ryzhik, I. M. (2007). Table of Integrals, Series, and Products, (7th ed.), Academic Press, San Diego.

Guardiola, J. H. (2004). The Semicircular Normal Distribution, Ph. D. Dissertation, Baylor University, Institute of Statistics.

Jammalamadaka, S. R. and SenGupta, A. (2001). Topics in Circular Statistics, World Scientific Publishing, Singapore.

Johnson, N. L. and Kotz, S. (1972). Continuous Multivariate Distributions, John Wiley \& Sons, New York.

Jones, T. A. (1968). Statistical analysis of orientation data, Journal of Sedimentary Petrology, 38, 61-67.

Kim, H. M. (2008). New family of the $t$ distributions for modeling semicircular data, Communications of the Korean Statistical Society, To appear.

Mardia, K. V. and Jupp, P. E. (2000). Directional Statistics, John Wiley \& Sons, Chichester.

Ugai, S., Kato, K., Nishijima, M. and Kan, T. (1977), Characteristics of raindrop size and raindrop shape, Open Symposium URSI Commission F, 225-230.

[Received July 2008, Accepted August 2008] 